\title{
SHARP HAMILTON'S LAPLACIAN ESTIMATE FOR THE HEAT KERNEL ON COMPLETE MANIFOLDS
}

\author{
JIA-YONG WU
}

(Communicated by Lei $\mathrm{Ni}$ )

\begin{abstract}
In this paper we give Hamilton's Laplacian estimates for the heat equation on complete noncompact manifolds with nonnegative Ricci curvature. As an application, combining Li-Yau's lower and upper bounds of the heat kernel, we give an estimate on Laplacian form of the heat kernel on complete manifolds with nonnegative Ricci curvature that is sharp in the order of time parameter for the heat kernel on the Euclidean space.
\end{abstract}

\section{INTRODUCTION}

In [4, R. Hamilton established the following gradient and Laplacian estimates for a bounded positive solution to the heat equation on closed manifolds with Ricci curvature bounded below.

Theorem 1.1 (R. Hamilton [4). Let $(M, g)$ be an n-dimensional closed Riemannian manifold with Ricci curvature satisfying Ric $\geq-K$ for some constant $K \geq 0$. Assume that $u$ is a positive solution to the heat equation with $u \leq A$ on $M \times[0, T]$ for some constant $A<\infty$, where $0<T<\infty$. Then

$$
t \frac{|\nabla u|^{2}}{u^{2}} \leq(1+2 K t) \log \left(\frac{A}{u}\right) .
$$

If we further assume $T \leq 1$, then for $0 \leq t \leq T$,

$$
t \frac{\Delta u}{u} \leq C(n, K)\left[1+\log \left(\frac{A}{u}\right)\right] .
$$

For estimate (1.2), when $K=0$, by choosing function $\varphi=t$ in Hamilton's proof of Lemma 4.1 in [4], we easily confirm that the condition " $T \leq 1$ " can be removed. Hamilton's estimate (1.1) shows that one can compare two different points at the same time. However the well-known Li-Yau's gradient estimate [7] only allows comparisons between different points at different times. In [6], B. Kotschwar generalized the gradient estimate (1.1) to the case of complete, noncompact Riemannian manifolds with Ricci curvature bounded below. Using this generalization, Kotschwar gave an estimate on the gradient of the heat kernel for complete manifolds with nonnegative Ricci curvature. Moreover, this estimate is sharp in the order of $t$ for the heat kernel on $\mathbb{R}^{n}$.

Received by the editors February 10, 2012.

2010 Mathematics Subject Classification. Primary 58J35; Secondary 35K08.

Key words and phrases. Gradient estimate, heat kernel, heat equation.

This work was partially supported by the NSFC (11101267, 11271132) and the Innovation Program of Shanghai Municipal Education Commission (13YZ087). 
Kotschwar's result can be used to prove the monotonicity of Ni's entropy functional (stationary metric for Perelman's $\mathcal{W}$-functional in [10]) in 8 for the fundamental solution to the heat equation on complete, noncompact manifolds. We would like to point out that in the course of justifying the monotonicity of Ni's entropy functional on complete noncompact manifolds, one may need a noncompact version of Hamilton's Laplacian estimate (1.2). However, as far as we know, perhaps no one has generalized the estimate (1.2) to the complete noncompact case. In [2, the authors only briefly sketched a proof of the estimate (1.2) for the noncompact case but didn't give any detail. In this paper, we will provide a full detailed proof that Hamilton's Laplacian estimate (1.2) also holds for complete, noncompact manifolds with nonnegative Ricci curvature. Precisely, we show that:

Theorem 1.2. Let $(M, g)$ be an n-dimensional complete noncompact Riemannian manifold with nonnegative Ricci curvature. Suppose $u$ is a smooth positive solution to the heat equation

$$
\frac{\partial u}{\partial t}-\Delta u=0
$$

satisfying $u \leq A$ for some constant $A<\infty$ on $M \times[0, T]$, where $0<T<\infty$. Then

$$
t \frac{\Delta u}{u} \leq n+4 \log \left(\frac{A}{u}\right)
$$

for all $x \in M$ and $0 \leq t \leq T$.

The proof of Theorem 1.2 is similar to the arguments of Kotschwar [6], which can be divided into two steps. In the first step, we obtain some Bernstein-type estimate of $\Delta u$, similar to the upper estimate of $|\nabla u|$ derived by Kotschwar [6] on complete noncompact manifolds. In the second step, using upper estimates of $\Delta u$ and $|\nabla u|$, we apply the maximum principle to the quantity of Hamilton's Laplacian estimate on complete noncompact Riemannian manifolds due to Karp-Ni [5] or NiTam 9]. We remark that the a priori integral bound needed for the application of the maximum principle on complete noncompact manifolds has also been obtained in 3 and in 13 for a more general setting.

As an application of Theorem 1.2 we obtain the following Laplacian estimate of the heat kernel on a complete noncompact Riemannian manifold with nonnegative Ricci curvature.

Theorem 1.3. Let $(M, g)$ be an n-dimensional complete noncompact Riemannian manifold with nonnegative Ricci curvature and $H(x, y, t)$ be its heat kernel. Then, for all $\delta>0$, there exists a constant $C=C(n, \delta)$ such that

$$
\frac{\Delta H}{H}(x, y, t) \leq \frac{2}{t}\left[C+4 \frac{d^{2}(x, y)}{(4-\delta) t}\right]
$$

for all $x, y \in M$ and $t>0$.

Remark 1.4. We would like to point out that Theorem 1.3 is sharp in the order of $t$ for the heat kernel on $\mathbb{R}^{n}$.

The structure of this paper is organized as follows. In Section 2, we derive Bernstein-type gradient estimates of the Laplacian for solutions to the heat equation (see Theorem 2.7). Our proof makes use of Shi's gradient estimates [1], combining the classical cut-off function arguments. In Section 3 we finish the proof 
of Theorem 1.2 by using Theorem 2.7. In Section 4, we apply Theorem 1.2 to the heat kernel and complete the proof of Theorem 1.3 .

\section{Bernstein-type estimates}

In this section, we assume that $(M, g)$ is an $n$-dimensional complete noncompact Riemannian manifold with the Ricci curvature uniformly bounded below by $-K$ for some constant $K \geq 0$ and suppose that $u$ is a smooth solution to the heat equation (1.3) satisfying $|u| \leq A$ on some open $U \subset M$ for $0 \leq t \leq T<\infty$. First we recall Kotschwar's result in $[$ ].

Theorem 2.1 (Kotschwar [6]). Let $(M, g)$ be an n-dimensional complete noncompact Riemannian manifold with Ric $\geq-K$ for some constant $K \geq 0$. Suppose $u$ is a smooth solution to the heat equation (1.3) satisfying $|u| \leq A$ on $B_{p}(2 R) \times[0, T]$ for some $p \in M^{n}$ and $A, R, T>0$. Then there exists a constant $C=C(n, K)$ such that

holds on $B_{p}(R) \times[0, T]$.

$$
t|\nabla u|^{2} \leq C A^{2}\left[1+T\left(1+\frac{1}{R^{2}}\right)\right]
$$

Remark 2.2. If Ric $\geq 0$, from the proof course of Theorem [2.1] in [6], one shows that

on $B_{p}(R) \times[0, T]$.

$$
t|\nabla u|^{2} \leq C(n) A^{2}\left(1+\frac{T}{R^{2}}\right)
$$

Remark 2.3. Letting $R \rightarrow \infty$ in the proof course of Theorem 2.1 one immediately shows that there exists a constant $C(n)$ such that

$$
t|\nabla u|^{2} \leq C(n) A^{2}(1+K T)
$$

on $M^{n} \times[0, T]$.

In the above description, Kotschwar showed the first derivative estimate of the positive solution to the heat equation on complete manifolds. Below we will give an upper estimate of $\Delta u$. Our proof is similar in spirit to the derivative estimates due to Shi [1] (see also 6]). Let

$$
F(x, t):=\left(C+t|\nabla u|^{2}\right) t^{2}|\Delta u|^{2},
$$

where the constant $C$ is to be chosen. The following lemma is useful for proving Theorem 2.7

Lemma 2.4. Let $(M, g)$ be an $n$-dimensional complete Riemannian manifold with Ric $\geq-K$ for some constant $K \geq 0$. If $0<u \leq A$ is the solution to the heat equation (1.3) on $B_{p}(2 R) \times[0, T]$ for some $p \in M$ and $A, R, T>0$, where $T \leq 1$, satisfying

$$
|\nabla u|^{2} \leq \frac{C_{*}}{t}
$$

for some constant $C_{*}$ on $B_{p}(R) \times(0, T]$, then there exists a finite positive constant $c:=c(n, K, A, R)$ such that

on $B_{p}(R) \times(0, T]$.

$$
\frac{\partial F}{\partial t} \leq \Delta F-\frac{c}{t} F^{2}+\frac{C_{*}^{2}}{t}
$$


Remark 2.5. The assumption $T \leq 1$ in Lemma 2.4 is used only in (2.3). By Theorem 2.1 one may choose $C_{*}:=C(n, K) A^{2}\left[1+T\left(1+\frac{1}{R^{2}}\right)\right]$. Also we can choose $c:=C^{-1}(n) \cdot C_{*}^{-2}$. Moreover, if $R \rightarrow \infty$, then $\lim _{R \rightarrow \infty} c$ is a finite positive constant. Note that here the constant $C(n, K)$ may be different from the one in Theorem 2.1

Remark 2.6. When $K=0$, from (2.3), we see that the assumption $T \leq 1$ can be replaced by $T<\infty$. In this case, from Remark 2.2, one can choose $C_{*}:=$ $C(n) A^{2}\left(1+\frac{T}{R^{2}}\right)$ and $c:=C^{-1}(n) \cdot C_{*}^{-2}$. If $R \rightarrow \infty$, then $\lim _{R \rightarrow \infty} c$ can still be a finite positive constant, independent on $T$.

Proof. At first, the evolution formula of $t|\nabla u|^{2}$ is

$$
\begin{aligned}
\left(\frac{\partial}{\partial t}-\Delta\right)\left(t|\nabla u|^{2}\right) & =-2 t|\nabla \nabla u|^{2}-2 t \operatorname{Ric}(\nabla u, \nabla u)+|\nabla u|^{2} \\
& \leq-t|\nabla \nabla u|^{2}-\frac{t}{n}|\Delta u|^{2}-2 t \operatorname{Ric}(\nabla u, \nabla u)+|\nabla u|^{2} \\
& \leq-t|\nabla \nabla u|^{2}-\frac{t}{n}|\Delta u|^{2}+(2 K t+1)|\nabla u|^{2}
\end{aligned}
$$

where we used Ric $\geq-K$. Then we compute that

$$
\left(\frac{\partial}{\partial t}-\Delta\right)|\Delta u|^{2}=-2|\nabla \Delta u|^{2}
$$

and hence

$$
\left(\frac{\partial}{\partial t}-\Delta\right)\left(t^{2}|\Delta u|^{2}\right)=-2 t^{2}|\nabla \Delta u|^{2}+2 t|\Delta u|^{2} .
$$

By the assumption of this lemma, we can choose $C$ in (2.2) such that $C=8 C_{*}$, which implies that $8 t|\nabla u|^{2} \leq C$. Combining the above equations yields

$$
\begin{aligned}
\left(\frac{\partial}{\partial t}-\Delta\right) F= & \left(C+t|\nabla u|^{2}\right)\left[\left(\frac{\partial}{\partial t}-\Delta\right)\left(t^{2}|\Delta u|^{2}\right)\right] \\
& +\left[\left(\frac{\partial}{\partial t}-\Delta\right)\left(C+t|\nabla u|^{2}\right)\right] t^{2}|\Delta u|^{2} \\
& -2 t^{3} \nabla\left(|\nabla u|^{2}\right) \cdot \nabla\left((\Delta u)^{2}\right) \\
\leq & \left(C+t|\nabla u|^{2}\right)\left(-2 t^{2}|\nabla \Delta u|^{2}+2 t|\Delta u|^{2}\right) \\
& +\left[-t|\nabla \nabla u|^{2}-\frac{t}{n}|\Delta u|^{2}+(2 K t+1)|\nabla u|^{2}\right] t^{2}|\Delta u|^{2} \\
& +8 t^{3}|\nabla u||\nabla \nabla u| \cdot|\Delta u||\nabla \Delta u| \\
\leq & -18 t^{3}|\nabla u|^{2}|\nabla \Delta u|^{2}-\frac{t^{3}}{n}(\Delta u)^{4}-t^{3}|\nabla \nabla u|^{2}(\Delta u)^{2} \\
& +2 t(\Delta u)^{2}\left(C+t|\nabla u|^{2}\right)+(2 K t+1) t^{2}|\nabla u|^{2}|\Delta u|^{2} \\
& +t^{3}|\nabla \nabla u|^{2} \cdot|\Delta u|^{2}+16 t^{3}|\nabla u|^{2} \cdot|\nabla \Delta u|^{2},
\end{aligned}
$$


where we used the Schwarz inequality. Since $t \leq T \leq 1$, the above formula becomes

$$
\begin{aligned}
\left(\frac{\partial}{\partial t}-\Delta\right) F & \leq-\frac{t^{3}}{n}(\Delta u)^{4}+(2 K t+1) C t|\Delta u|^{2}+4 C t(\Delta u)^{2} \\
& \leq-\frac{t^{3}}{n}(\Delta u)^{4}+(2 K+1) C t|\Delta u|^{2}+4 C t(\Delta u)^{2} \\
& \leq-\frac{c}{t} F^{2}+\frac{18 n\left(1+K^{2}\right) C^{2}}{t}
\end{aligned}
$$

where in the last inequality we used

$$
\frac{t^{3}}{2 n}(\Delta u)^{4}+\frac{18 n\left(1+K^{2}\right) C^{2}}{t} \geq C(2 K+1) t|\Delta u|^{2}+4 C t(\Delta u)^{2} .
$$

Here $c:=c(n, K, A, R)$ depends on $n, K, A$ and $R$. For example, we may choose $c:=C^{-1}(n) \cdot C_{*}^{-2}$. Then the result follows.

Using Lemma 2.4, we prove the following Laplacian estimate for the positive solution to the heat equation.

Theorem 2.7. Let $(M, g)$ be an $n$-dimensional complete Riemannian manifold with Ric $\geq-K$ for some constant $K \geq 0$. Let $u$ be a positive solution to the heat equation (1.3) with $u \leq A$ on $B_{p}(2 R) \times(0, T]$ for some $p \in M^{n}$ and $A, R, T>0$, where $A<\infty$ and $T \leq 1$. Then there exists a constant $C=C(n, K)$ such that

$$
t|\Delta u| \leq C A\left[1+T\left(1+\frac{1}{R^{2}}\right)\right]^{1 / 2} \cdot\left(1+\frac{T}{R^{2}}\right)^{1 / 2}
$$

on $B_{p}(R) \times[0, T]$.

Remark 2.8. When $K=0$, the assumption $T \leq 1$ can be replaced by $T<\infty$. In this case, from Remark 2.6] estimate (2.4) can be rewritten by a simple version:

$$
t|\Delta u| \leq C(n) A\left(1+\frac{T}{R^{2}}\right)
$$

on $B_{p}(R) \times[0, T]$. If we further let $R \rightarrow \infty$, then

$$
t|\Delta u| \leq C(n) A
$$

on $M \times[0, T]$.

Proof of Theorem 2.7. As in [1,7] (see also [6] or [12), for any $p \in M$ and $R>0$, we may choose a cut-off function with $\eta(x)=1$ on $B_{p}(R)$ and supported in $B_{p}(2 R)$ satisfying

$$
|\nabla \eta|^{2} \leq \frac{C_{3}}{R^{2}} \eta
$$

and

$$
\Delta \eta \geq-\frac{C_{3}}{R^{2}}
$$

for some $C_{3}=C_{3}(n)>0$. Letting $G:=\eta F$, we compute that

$$
\left(\frac{\partial}{\partial t}-\Delta\right) G \leq \frac{\eta}{t}\left(-c F^{2}+C_{*}^{2}\right)-\Delta \eta \cdot F-2 \nabla \eta \cdot \nabla F
$$


where $C_{*}=C(n, K) A^{2}\left[1+T\left(1+\frac{1}{R^{2}}\right)\right]$. Assume that at a point $\left(x_{0}, t_{0}\right)$ the function $G$ attains its positive maximum in $B(p, 2 R) \times(0, T]$. Then at $\left(x_{0}, t_{0}\right)$ we have

$$
\left(\frac{\partial}{\partial t}-\Delta\right) G \geq 0 \quad \text { and } \quad 0=\nabla G=\eta \nabla F+F \nabla \eta
$$

Therefore at $\left(x_{0}, t_{0}\right)$ we have

$$
\begin{aligned}
0 & \leq \frac{1}{t}\left[-c(\eta F)^{2}+C_{*}^{2} \eta\right]-\Delta \eta \cdot(\eta F)+2 F|\nabla \eta|^{2} \\
& \leq \frac{1}{t}\left[-c G^{2}+C_{*}^{2} \eta\right]+\frac{C_{3}}{R^{2}} G+2 \frac{C_{3}}{R^{2}} G
\end{aligned}
$$

and hence

$$
c G^{2}\left(x_{0}, t_{0}\right) \leq C_{*}^{2}+3 \frac{C_{3}}{R^{2}} G\left(x_{0}, t_{0}\right) t_{0}
$$

Since

$$
3 \frac{C_{3}}{R^{2}} G\left(x_{0}, t_{0}\right) t_{0} \leq \frac{c}{2} G^{2}\left(x_{0}, t_{0}\right)+\frac{8 C_{3}^{2}}{c R^{4}} t_{0}^{2},
$$

inequality (2.9) implies that

$$
\frac{c}{2} G^{2}\left(x_{0}, t_{0}\right) \leq C_{*}^{2}+\frac{8 C_{3}^{2}}{c R^{4}} t_{0}^{2} .
$$

Therefore for any $(x, t) \in B_{p}(R) \times[0, T]$,

$$
G^{2}(x, t) \leq G^{2}\left(x_{0}, t_{0}\right) \leq 2 c^{-1} C_{*}^{2}+c^{-2} \frac{16 C_{3}^{2}}{R^{4}} t_{0}^{2} .
$$

Since $c:=C^{-1}(n) \cdot C_{*}^{-2}$, we have that

$$
G^{2}(x, t) \leq C(n) C_{*}^{4}+\frac{C(n)}{R^{4}} T^{2} C_{*}^{4}
$$

for any $(x, t) \in B_{p}(R) \times[0, T]$. This implies

$$
G(x, t) \leq C(n) C_{*}^{2}+\frac{C(n)}{R^{2}} T C_{*}^{2}
$$

for any $(x, t) \in B_{p}(R) \times[0, T]$. By the definitions of $C_{*}$ and $G$, we have

$$
8 C_{*} t^{2}|\Delta u|^{2} \leq C(n) C_{*}^{2}+\frac{C(n)}{R^{2}} T C_{*}^{2},
$$

and therefore

$$
t^{2}|\Delta u|^{2} \leq C(n, K) A^{2}\left[1+T\left(1+\frac{1}{R^{2}}\right)\right] \cdot\left(1+\frac{T}{R^{2}}\right)
$$

for any $(x, t) \in B_{p}(R) \times[0, T]$, which completes the proof of the theorem.

\section{Proof of Theorem 1.2}

In this section, by using gradient and Laplacian estimates of the previous section, we apply a maximum principle on complete noncompact manifolds due originally to Karp and Li [5] (see also Ni-Tam [9]) to finish the proof of Theorem 1.2. 
Theorem 3.1 (Karp-Li [5] and Ni-Tam [9]). Let $(M, g)$ be an n-dimensional complete Riemannian manifold. Suppose $f(x, t)$ is a smooth function on $M \times[0, T]$, $0<T<\infty$, such that

$$
\left(\frac{\partial}{\partial t}-\Delta\right) f(x, t) \leq 0 \quad \text { whenever } \quad f(x, t) \leq 0 .
$$

Let $f_{+}(x, t):=\max \{f(x, t), 0\}$. Assume that

$$
\int_{0}^{T} \int_{M} e^{-a r^{2}(x)} f_{+}^{2}(x, t) d \mu d t \leq 0
$$

for some constant $a>0$, where $r(x)$ is the distance to $x$ from some fixed $p \in M$. If $f(x, 0) \leq 0$ for all $x \in M$, then $f(x, t) \leq 0$ for all $(x, t) \in M \times[0, T]$.

Proof of Theorem 1.2. Following Hamilton's proof [4] with a little modification, we define $u_{\epsilon}=u+\epsilon$ satisfying $\epsilon<u_{\epsilon}<A+\epsilon$ and the function

$$
P(x, t):=t\left(\Delta u_{\epsilon}+\frac{\left|\nabla u_{\epsilon}\right|^{2}}{u_{\epsilon}}\right)-u_{\epsilon}\left(n+4 \log \frac{A}{u_{\epsilon}}\right) .
$$

By 4, we have

$$
\left(\frac{\partial}{\partial t}-\Delta\right) P \leq-\frac{2 t}{n u_{\epsilon}}\left(\Delta u_{\epsilon}-\frac{\left|\nabla u_{\epsilon}\right|^{2}}{u_{\epsilon}}\right)^{2}+\left(\Delta u_{\epsilon}-\frac{\left|\nabla u_{\epsilon}\right|^{2}}{u_{\epsilon}}\right)-2 \frac{\left|\nabla u_{\epsilon}\right|^{2}}{u_{\epsilon}} .
$$

By Hamilton's arguments, we easily have that

$$
\left(\frac{\partial}{\partial t}-\Delta\right) P \leq 0 \quad \text { whenever } \quad P \geq 0
$$

This fact can be obtained by the following three cases:

(1) If $\Delta u_{\epsilon} \leq \frac{\left|\nabla u_{\epsilon}\right|^{2}}{u_{\epsilon}}$, then we are done.

(2) If $\frac{\left|\nabla u_{\epsilon}\right|^{2}}{u_{\epsilon}} \leq \Delta u_{\epsilon} \leq 3 \frac{\left|\nabla u_{\epsilon}\right|^{2}}{u_{\epsilon}}$, then we are also done.

(3) If $3 \frac{\left|\nabla u_{\epsilon}\right|^{2}}{u_{\epsilon}} \leq \Delta u_{\epsilon}$, then when $P \geq 0$, we have

$$
2\left(\Delta u_{\epsilon}-\frac{\left|\nabla u_{\epsilon}\right|^{2}}{u_{\epsilon}}\right) \geq \Delta u_{\epsilon}+\frac{\left|\nabla u_{\epsilon}\right|^{2}}{u_{\epsilon}} \geq \frac{n u_{\epsilon}}{t},
$$

and hence we are completely done.

Now, obviously, we have

$$
P(x, 0)<0 .
$$

By our assumptions on $u_{\epsilon}$, we also have

$$
P_{+}(x, t) \leq t\left(\Delta u_{\epsilon}+\frac{1}{\epsilon}\left|\nabla u_{\epsilon}\right|^{2}\right),
$$

where $P_{+}(x, t):=\max \{P(x, t), 0\}$. Thus, using estimates (2.1) and (2.6), for any $p \in M^{n}$ and $T, R>0$, we have

$$
\begin{aligned}
\int_{0}^{T} \int_{B_{p}(R)} & e^{-r^{2}(x)} P_{+}^{2}(x, t) d \mu d t \\
& \leq \int_{0}^{T} \int_{B_{p}(R)} e^{-r^{2}(x)}\left[t\left(\Delta u_{\epsilon}+\frac{1}{\epsilon}\left|\nabla u_{\epsilon}\right|^{2}\right)\right]^{2} d \mu d t \\
& \leq\left(C(n) A+\frac{C(n) A^{2}}{\epsilon}\right)^{2} \int_{0}^{T} \int_{M} e^{-r^{2}(x)} d \mu d t .
\end{aligned}
$$


Since Ric $\geq 0$, by the Bishop volume comparison theorem, we have that

$$
\int_{0}^{T} \int_{B_{p}(R)} e^{-r^{2}(x)} d \mu d t<\infty
$$

Then by letting $R \rightarrow \infty$, we conclude that

$$
\int_{0}^{T} \int_{M} e^{-r^{2}(x)} d \mu d t<\infty
$$

and hence

$$
\int_{0}^{T} \int_{B_{p}(R)} e^{-r^{2}(x)} P_{+}^{2}(x, t) d \mu d t<\infty .
$$

By the maximum principle for the complete noncompact manifold, we conclude that $P(x, t) \leq 0$ for all $t \leq T$, and hence the conclusion of Theorem 1.2 follows.

\section{Proof of Theorem 1.3}

The proof of Theorem 1.3 follows from that of Theorem 1 in [6] with little modification, but is included for completeness.

Proof of Theorem 1.3. Let $H(x, y, t)$ be the heat kernel of the heat equation on $(M, g)$. For any $t>0$ and $y \in M$, we set $u(x, s):=H(x, y, s+t / 2)$, and then $u$ is a smooth, positive solution to the heat equation on [0,T). By [7], for any $\delta>0$ there exists a constant $C_{1}=C_{1}(\delta)>0$ such that

$$
\frac{\exp \left(\frac{-d^{2}(x, y)}{(4-\delta)(s+t / 2)}\right)}{C_{1} \operatorname{Vol}\left(B_{y}(\sqrt{s+t / 2})\right)} \leq u(x, s) \leq \frac{C_{1}}{\operatorname{Vol}\left(B_{y}(\sqrt{s+t / 2})\right)}
$$

for all $x, y \in M$, and $s \geq 0$.

Letting

$$
A:=\frac{C_{1}}{\operatorname{Vol}\left(B_{y}(\sqrt{t / 2})\right)},
$$

the latter part of inequality (4.1) implies $u \leq A$ for all $x$ and $s$. Since Ric $\geq 0$, there exists a positive constant $C_{2}:=C_{2}(n)$ such that

$$
\operatorname{Vol}\left(B_{y}(\sqrt{s+t / 2})\right) \leq \operatorname{Vol}\left(B_{y}(\sqrt{t})\right) \leq C_{2} \operatorname{Vol}\left(B_{y}(\sqrt{t / 2})\right)
$$

for all $0 \leq s \leq t / 2$. Thus, by the front part of inequality (4.1) and Theorem 1.2. we have

$$
s \frac{\Delta u}{u} \leq n+4 \log \left(\frac{A}{u}\right) \leq n+4 \log \left(C_{1}^{2} C_{2}\right)+\frac{4 d^{2}(x, y)}{(4-\delta)(s+t / 2)}
$$

on $M \times[0, t / 2]$. Setting $C=n+4 \log \left(C_{1}^{2} C_{2}\right)$ and $s=t / 2$ from above, we conclude that

$$
(t / 2) \frac{\Delta H}{H}(x, y, t)=(t / 2) \frac{\Delta u}{u}(x, t / 2) \leq C+4 \frac{d^{2}(x, y)}{(4-\delta) t}
$$

for all $x, y \in M$ and $t>0$.

\section{ACKNOWLEDGMENT}

The author would like to express his gratitude to the referee for careful readings and many valuable suggestions. 


\section{REFERENCES}

[1] E. Calabi, An extension of E. Hopf's maximum principle with an application to Riemannian geometry, Duke Math. J. 25 (1958), 45-56. MR0092069 (19,1056e)

[2] Bennett Chow, Sun-Chin Chu, David Glickenstein, Christine Guenther, James Isenberg, Tom Ivey, Dan Knopf, Peng Lu, Feng Luo, and Lei Ni, The Ricci flow: techniques and applications. Part II. Analytic aspects, Mathematical Surveys and Monographs, vol. 144, American Mathematical Society, Providence, RI, 2008. MR2365237 (2008j:53114)

[3] Alexander Grigor'yan, Upper bounds of derivatives of the heat kernel on an arbitrary complete manifold, J. Funct. Anal. 127 (1995), no. 2, 363-389, DOI 10.1006/jfan.1995.1016. MR 1317722 (96a:58183)

[4] Richard S. Hamilton, A matrix Harnack estimate for the heat equation, Comm. Anal. Geom. 1 (1993), no. 1, 113-126. MR1230276 (94g:58215)

[5] L. Karp, P. Li, The heat equation on complete Riemannian manifolds, http://math. uci.edu/ $\sim$ pli/, preprint, 1982.

[6] Brett L. Kotschwar, Hamilton's gradient estimate for the heat kernel on complete manifolds, Proc. Amer. Math. Soc. 135 (2007), no. 9, 3013-3019 (electronic), DOI 10.1090/S0002-993907-08837-5. MR2317980(2008e:58034)

[7] Peter Li and Shing-Tung Yau, On the parabolic kernel of the Schrödinger operator, Acta Math. 156 (1986), no. 3-4, 153-201, DOI 10.1007/BF02399203. MR834612 (87f:58156)

[8] Lei Ni, Addenda to: "The entropy formula for linear heat equation" [J. Geom. Anal. 14 (2004), no. 1, 87-100; MR 2030576], J. Geom. Anal. 14 (2004), no. 2, 369-374, DOI 10.1007/BF02922078. MR2051693(2004m:53118b)

[9] Lei Ni and Luen-Fai Tam, Kähler-Ricci flow and the Poincaré-Lelong equation, Comm. Anal. Geom. 12 (2004), no. 1-2, 111-141. MR2074873 (2005f:53108)

[10] G. Perelman, The entropy formula for the Ricci flow and its geometric applications, arXiv:math.DG/0211159v1, 2002.

[11] Wan-Xiong Shi, Deforming the metric on complete Riemannian manifolds, J. Differential Geom. 30 (1989), no. 1, 223-301. MR.1001277(90i:58202)

[12] Jia-Yong Wu, Li-Yau type estimates for a nonlinear parabolic equation on complete manifolds, J. Math. Anal. Appl. 369 (2010), no. 1, 400-407, DOI 10.1016/j.jmaa.2010.03.055. MR2643878(2011b:35432)

[13] Chengjie Yu, Some estimates of fundamental solutions on noncompact manifolds with timedependent metrics, Manuscripta Math. 139 (2012), no. 3-4, 321-341, DOI 10.1007/s00229011-0517-y. MR2974280

Department of Mathematics, Shanghai Maritime University, Haigang Avenue 1550, Shanghai 201306, People's Republic of China

E-mail address: jywu81@yahoo.com 